\title{
PROSUMO, ALIENACIÓN Y EXPLOTACIÓN. REFLEXIONES EN TORNO AL TRABAJO DIGITAL
}

\author{
Prosumption, alienation an exploitation. Reflections around digital labour \\ Mikel Barba Del Horno* \\ Universidad del País Vasco/Euskal Herriko Unibertsitatea, España
}

\begin{abstract}
RESUMEN
Las transformaciones digitales están teniendo una gran influencia en el ámbito del trabajo. El sociólogo George Ritzer ha propuesto una gran narrativa que defiende que estamos entrando en una nueva fase del capitalismo que él denomina capitalismo de prosumidores. En este nuevo estadio se estaría produciendo una creciente confusión entre las actividades de producción y consumo. Desde una perspectiva marxista, Christian Fuchs ha defendido, basándose en la Teoría del Valor Trabajo de Marx, que los prosumidores son trabajadores explotados.

Desde el punto de vista de muchos autores y autoras, en la era digital se estarían produciendo transformaciones importantes en los procesos de trabajo que estarían alterando la misma dinámica de acumulación del capital. En este artículo se presenta una propuesta teórica alternativa para tratar de comprender los nuevos fenómenos del trabajo digital que está basada en los conceptos de alienación y explotación y que defiende una continuidad con los procesos de transformación del trabajo que se encuentra en marcha desde los años setenta.

Palabras clave: trabajo digital, prosumidor, explotación, alienación.

\section{ABSTRACT}

Digital transformations are having a great influence in the field of labour. Sociologist George Ritzer has proposed a grand narrative arguing that we are entering a new phase of capitalism that he calls prosumer capitalism. In this new stage, there is a growing confusion between production and consumption activities. From a Marxist perspective, Christian Fuchs has argued, based on Marx's Labor Theory of Value, that prosumers are exploited workers.

According to many authors, in the digital age, important transformations in work processes would be taking place, and these transformations would be altering the dynamics of capital accumulation. This article presents an alternative proposal to understand the new phenomena of digital work, based on the concepts of alienation and exploitation, that defends a continuity with the labour transformation processes that started in the 1970 s.

Keywords: digital labour, prosumer, exploitation, alienation.
\end{abstract}

\section{LABURPENA}

Berrikuntza digitalak lan arloan aldaketa handiak eragiten ditu. Honen harira, George Ritzer soziologoak narratiba handi bat proposatu du. Bere ustez, prosumer kapitalismoa bezala izendatzen duen etapa berri batean gaude murgilduta. Aro honetan, ekoizpen eta kontsumo jarduerak modu nahasian agertzen zaizkigu. Ikuspuntu marxista batetik, Christian Fuchs-ek prosumer-ak langile esplotatuak direla defendatu egin du, Marx-en lan balioaren teoria abiapuntu bezala erabilita.

Egile askoren arabera, aro digitalean lanaren eraldaketa nabarmena gauzatzen ari da eta, honek, kapitalaren akumulazioaren dinamika bera aldatu egiten du. Artikulu honetan lan digitalaren fenomenoa ulertzeko proposamen teoriko alternatibo bat aurkezten da. Alienazio eta esplotazio konteptuetatik abiatuta hirurogei ta hamarreko hamarkadetan hasi ziren lanaren eraldaketa dinamikekin jarraitutasuna dagoela defendatzen da.

Hitz Gakoak: lan digitala, prosumer, esplotazioa, alienazioa.

* Correspondencia a: Mikel Barba Del Horno. Paseo Santsoena Auzoa, 26, Leioa (Bizkaia), España. — mikel.barba@ehu. eus - https: //orcid.org/0000-0002-4261-9843

Cómo citar: Barba Del Horno, Mikel. (2021). «Prosumo, alienación y explotación. Reflexiones en torno al trabajo digital»; Lan Harremanak, 45, 184-207. (https: //doi.org/10.1387/lan-harremanak.22812).

Recibido: 15 mayo, 2021; aceptado: 09 junio, 2021.

ISSN 1575-7048 - eISSN 2444-5819 / (c) 2020 UPV/EHU

(c) (i) Esta obra está bajo una licencia

Creative Commons Atribución 4.0 Internacional 


\section{Introducción}

$\mathrm{El}$ advenimiento de internet ha dado lugar a transformaciones importantes en el ámbito del trabajo. En los últimos tiempos, empresas como Uber, Cabify o Amazon a través de su proyecto del Mechanical Turk han sido vistos como graves amenazas para el sostenimiento de los derechos laborales. Los trabajadores de estas plataformas tienen por lo general trabajos más precarios, peor pagados, con requisitos de cualificación inferiores y con horarios flexibles en los que tienen un mayor peso de lo normal el trabajo nocturno y en festivos (OIT, 2019).

Además de esta tendencia a la precarización algunos autores han observado un proceso de creciente indefinición de la actividad laboral. Se ha recuperado para dar cuenta de ello un concepto que popularizó Alvin Toffler (1985) en los años 80; el prosumidor. Este neologismo que se crea a través de la contracción de las palabras productor y consumidor pretende dar cuenta de la creciente interrelación entre consumo y producción que provoca que, en ocasiones, sea difícil, si no imposible, distinguir ambos procesos. El concepto de prosumidor se ha erigido como uno de los constructos principales para explicar las transformaciones del trabajo en la era digital.

En este trabajo se hace una reflexión teórica en torno al concepto de prosumidor y su capacidad para dar cuenta de los procesos de transformación del trabajo. Comenzaremos analizando dos de las aportaciones centrales en torno a la teorización del prosumo en el capitalismo digital: la de George Ritzer y la de Christian Fuchs. Desarrollaremos la idea de que existe un abuso del concepto, en el sentido de que se ha aplicado a dinámicas laborales y económicas que pueden ser mejor comprendidas a través de otros conceptos con un mayor recorrido. La ludificación del trabajo y la extensión del prosumo como formas de organización laboral, aun teniendo cierta importancia, no deberían de eclipsar la continuidad que existe con etapas anteriores del capitalismo. Siguiendo esta idea, se expone un modelo de análisis alternativo del trabajo digital en base a los conceptos de alienación y explotación.

El trabajo se estructura de la siguiente manera. En el primer apartado se repasa la propuesta teórica del capitalismo de prosumidores de George Ritzer. En el segundo se revisan algunos enfoques marxistas del concepto de prosumidor. En el tercer apartado se propone un esquema teórico alternativo basado en los conceptos de alienación y explotación que plantea la continuidad con las dinámicas de transformación del trabajo que se inician con la crisis del petróleo y las revoluciones del 68. En el cuarto apartado se aplica el esquema anterior a una tipología de trabajos digitales.

\section{Hacia un capitalismo de prosumidores: la propuesta de George Ritzer}

Una de las principales referencias en la investigación en torno al trabajo digital y el fenómeno de los prosumidores es el trabajo del sociólogo George Rit- 
zer (2014, 2015a, 2015b). Este autor había trabajado anteriormente en el análisis de las transformaciones del trabajo basadas en modelos de racionalización de la producción, del que los restaurantes McDonald's eran el ejemplo arquetípico (Ritzer, 1996).

En esta serie de nuevos trabajos Ritzer aborda el análisis de lo que denomina el capitalismo de prosumidores. Como punto de partida, propone superar la división binaria entre producción y consumo y partir de la idea de que ambos son tipos ideales en el sentido weberiano y, por lo tanto, no aparecen en forma pura en el mundo real. En la realidad, consumo y producción son fenómenos que no aparecen nítidamente separados; las actividades de producción implican siempre el consumo de bienes mientras que, cuando consumimos, estamos también contribuyendo a la producción de lo consumido en la medida que incorporamos trabajo para que el producto sea utilizable.

Así, según Ritzer, las trabajadoras de una cadena de producción que emplean máquinas y materias primas estarían, además de produciendo un bien, consumiendo esos insumos en el proceso productivo. El consumidor de un producto, por su parte, realiza actividades de producción porque tiene que desplazarse para adquirir el producto llevarlo a su casa, abrir el paquete, etc. Como plantearemos más adelante, el problema de partir de una distinción entre consumo y producción basada en la actividad en sí puede dar lugar a una confusión entre los conceptos que efectivamente hace imposible distinguirlos.

En cualquier caso, Ritzer parte de que consumo y producción no existen de manera pura en la realidad y que estaríamos siempre ante diferentes tipos de prosumo; combinaciones variables con mayores o menores cantidades de producción y consumo. Establece así que las actividades de prosumo pueden situarse en un continuo en cada uno de cuyos extremos estaría una forma típico ideal. En el extremo más cercanos a la producción pura se situaría lo que Ritzer denomina prosumo-como-producción (p-c-p), mientras que en el extremo más cercano al consumo puro tendríamos el prosumo-como-consumo (p-c-c).

Ritzer defiende que, aunque los conceptos de producción y consumo eran parcialmente válidos en la era del capitalismo industrial, en las últimas décadas se han convertido en conceptos zombie (Beck, 2001); conceptos que seguimos utilizando por inercia pero que han perdido su capacidad para explicar la realidad. En la era de lo que Ritzer denomina el capitalismo prosumidor están ganando importancia actividades que escapan claramente de la dicotomía producción-consumo; Ritzer pone como ejemplo el modelo de autoservicio, las plataformas de internet y los nuevos negocios como Uber que conectan el mundo material con el digital.

Ritzer afirma que la predominancia del prosumo en el capitalismo actual nos sitúa en una nueva época. Nos propone, de esta manera, una gran narrativa 
que define al capitalismo como un sistema que ha ido evolucionando históricamente desde un predominio de la producción hasta un predominio del prosumo. Ritzer distingue aquí tres estadios diferentes del sistema capitalista en los países desarrollados.

El capitalismo de producción se iniciaría con la industrialización y duraría hasta la Segunda Guerra Mundial. Esta etapa se caracteriza por el dominio de la producción y por el tipo de explotación centrada en el proceso de producción que Marx describió en el primer volumen de El Capital (Marx, 1981). La explotación del trabajo se basa en la extracción de la plusvalía, a saber, la parte del trabajo realizado por las personas trabajadoras que no les es remunerado.

La segunda etapa, el capitalismo de consumo, iría desde la Segunda Guerra Mundial hasta el fin del siglo xx, y estaría caracterizada por el predominio del consumo y el desarrollo de toda una serie de tecnologías orientadas a estimular la demanda. En este segundo periodo adquiere importancia la explotación de los consumidores basada en el poder de mercado de las empresas. Este poder se obtiene mediante la diferenciación del producto y el uso masivo de la mercadotecnia. Las empresas buscan crear necesidades artificiales en los individuos para que estos compren objetos que no necesitan y pagen precios superiores a los que corresponderían a los costes. Emerge de esta manera la figura del hiperconsumidor (Lipovetsky, 2010), que da sentido a su vida a través de los procesos de compra. En el capitalismo de consumo sigue existiendo la explotación ligada a la producción que conviviría con la explotación centrada en el consumo.

La tercera etapa, comienza en el siglo XXI cuando emerge un nuevo tipo de capitalismo, al que Ritzer denomina capitalismo prosumidor. Esta nueva etapa estaría vinculada a la revolución tecnológica que permite una creciente fusión entre los procesos de producción y consumo. En el capitalismo prosumidor la doble explotación de la etapa anterior se vuelve sinérgica; se dan los dos tipos de explotación en la misma actividad y al mismo tiempo. Las tecnologías de explotación del trabajo y del consumo se emplean también de manera sinérgica; de tal modo que el diseño tecnológico está orientado al ahorro de costes laborales no sólo aumentando la productividad de los trabajadores, sino también trasladando parte del trabajo a realizar a los consumidores, los p-a-c en términos de Ritzer.

Algunos opinan que los p-a-cs no están explotados porque obtienen un precio menor. Ritzer es crítico con esta idea. La explotación sinérgica no es un winwin. La tecnología la articulan las empresas con el objetivo fundamental de ahorrar costes laborales. Estas empresas tienen información sobre los costes que se ahorran con cada implementación y pueden ir modulando la tecnología para minimizar esos costes. Los prosumidores, por el contrario, carecen de la información sobre la cantidad de trabajo que están ahorrando las empresas y no saben si están siendo justamente recompensados por su labor. 
Según Ritzer, aunque parece que los p-a-c no están alienados porque no muestran un rechazo hacia la actividad de prosumo, teóricamente estarían tan alienados como los p-a-p; están separados del proceso de consumo y de otros pa-cs. La implicación principal de la implantación de estas tecnologías es que la explotación es mucho menos transparente en el caso de los p-a-c que en los pa-p; se da de una manera que dificulta que sea percibida por parte de los agentes. Ésta es una crítica bastante común a los procesos digitales, por cuanto se supone que ocultan la realidad; los algoritmos han sido descritos como cajas negras que automatizan procesos sociales, ocultándolos además a los ojos de la mayoría de las personas (Pasquale, 2015).

Por lo tanto, los p-a-c estarían doblemente explotados y, además, no serían conscientes de ello porque su explotación se encuentra oculta tras un velo tecnológico. Ritzer propone aquí que se está produciendo crecientemente fenómenos que podría colocarse bajo la categoría de hiperprosumo, individuos que se ven crecientemente empujados a realizar actividades de prosumo como una forma de definir su identidad.

Ritzer ha respondido a aquellos que consideran que no existe explotación en el caso de los prosumidores. Estos críticos consideran que los prosumidores realizan el trabajo porque ellos quieren sin que exista ningún tipo de coerción ni la necesidad de vender su fuerza de trabajo para subsistir como en el caso de los proletarios asalariados. Ritzer opina que los prosumidores están siendo explotados porque no son conscientes de estar realizando un trabajo del que la empresa se apropia. Se trata del mismo razonamiento del velo tecnológico al que aludíamos anteriormente.

Quizás en este punto exista una tendencia a sobrevalorar la capacidad de los algoritmos de ocultar los procesos sociales; no podemos perder de vista que la mayoría de la gente que usa redes sociales es consciente de que están recopilan datos que se usarán con fines comerciales, aunque desconozcan el uso concreto que se les va a dar a dichos datos. La preocupación por la privacidad es un problema político al que se presta atención y que ha dado lugar a la creación de una cantidad importante de regulaciones en los últimos ańos. Por otro lado, se tiende a minusvalorar otro tipo de mecanismos de ocultamiento y legitimación de las relaciones de poder que existían antes del algoritmo y que tienen un carácter más cultural que tecnológico.

En cualquier caso, Ritzer cree que el capitalismo de prosumidores va más allá de las dinámicas relacionadas con el fetichismo de la mercancía y va a dar lugar progresivamente a un sistema donde no será necesario el control ni la coerción y los trabajadores harán el trabajo porque lo desean. Como no existe conciencia del proceso de prosumo y este se puede activar en cualquier lugar y momento, la explotación se puede extender a las 24 horas del día. Ritzer piensa también que este modelo tiene ventajas claras para las corporaciones, que tienen la posibilidad de ahorrar trabajo, lo que llevará a una progresiva extensión del modelo. 
El trabajo de Ritzer resulta interesante, pero podrían hacérsele varias críticas. En primer lugar, aunque Ritzer plantea la cuestión de la gran narrativa en un sentido weberiano, es decir, como una herramienta analítica más que como una realidad esencial, no deja de caer en un cierto epocalismo tecnológico (Morozov, 2012). Esta es una acusación que Eugeni Morozov ha vertido sobre muchos de los analistas de internet y que, básicamente, denuncia una tendencia a sobrevalorar los cambios sociales asociados a la emergencia de nuevas pautas tecnológicas y a minusvalorar la resistencia al cambio que ofrecen las dinámicas estructurales. Existe, desde este punto de vista, una tendencia a sustituir las categorías analíticas vigentes por otras nuevas que parecerían adaptarse mejor a la realidad empírica. Sin embargo, esto no es siempre así; la realidad social tiene dimensiones estructurales que presentan una importante resistencia al cambio, por lo que gran parte de los conceptos que se utilizaban hace uno o dos siglos siguen conservando un poder explicativo importante. Como veremos más adelante, el empleo del concepto de prosumidor como concepto articulador, lejos de facilitar una mejor compresión teórica de los procesos de trabajo- producción y consumo puede contribuir a crear una mayor confusión.

\section{Marxismos digitales: a vueltas con la teoría del valor}

Otro trabajo interesante en torno al concepto de prosumidor y el trabajo digital es el que ha realizado desde el campo marxista Christian Fuchs. Fuchs ha tratado de recuperar las dimensiones comunicacionales que él cree que son centrales en el trabajo de Marx y que, según su punto de vista, han sido abandonadas por la mayoría de los teóricos marxista (Fuchs, 2019). Su teorización sobre el trabajo digital parte de la Teoría del Valor Trabajo (TVT) marxista y, por lo tanto, de la idea de que la extracción de la plusvalía se produce fundamentalmente durante el proceso de producción.

A diferencia de Ritzer, Fuchs considera que el capitalismo digital emerge como una suerte de subsistema que se integra en la unidad dialéctica mayor que constituye la totalidad del sistema capitalista. No existiría, desde este punto de vista, una ruptura con un capitalismo anterior. De hecho, Fuchs trata de explicar las dinámicas del capitalismo digital a partir de las categorías marxista tradicionales y las aplica a un nuevo tipo de trabajo, el trabajo digital.

Fuchs propone una tipología de trabajos digitales que se articulan en una División Internacional de Trabajo Digital (Fuchs, 2014). Describe así una serie de actividades insertas en contextos institucionales diversos y articuladas a través de diversas formas de organización del trabajo. La tipología abarcaría desde la extracción de los minerales necesarios para fabricar los dispositivos electrónicos hasta las tareas de diseño y programación que se desarrollan en Silicon Valley. 
El punto de partida lo constituyen los trabajadores en condiciones de esclavitud que extraen minerales como el coltán en el Congo. Un segundo caso sería el de China, donde tendríamos procesos que son muy similares a los que se produjeron en la época de los cercamientos en Inglaterra, con unas masas ingentes de población que están siendo expulsadas del ámbito rural y acaban trabajando en las fábricas de ensamblaje de productos electrónicos. En el caso de la India se daría un nuevo tipo de imperialismo orientado a explotar una mano de obra cualificada, la de los trabajadores del software, a unos costes laborales muy inferiores a los de los países del centro. La división internacional del trabajo se hace presente también en los países desarrollados a través de tres modalidades diferentes. Una sería la de los trabajadores precarios de los call centers que respondería a una organización taylorista del trabajo basada en el control. Fuchs recurre también, al concepto marxista de aristocracia obrera para referirse a los trabajadores cualificados y altamente remunerados de empresas como Google. La tercera categoría correspondería a los prosumidores; usuarios de redes sociales que en su proceso de consumo de los productos comunicacionales que ofrecen las plataformas están realizando un trabajo de producción de datos y están, por lo tanto, generando un valor. Como no reciben remuneración alguna por este trabajo los prosumidores están generando una plusvalía y, en consecuencia, están siendo explotados.

Uno de los debates del marxismo que recupera Fuchs en este punto es el que distingue entre trabajo productivo, el que genera valor a través de la producción de mercancías, y trabajo improductivo, el que sirve para sostener el sistema, pero que no genera valor (Fuchs, 2015). Desde una visión ortodoxa de la teoría del valor, quizás la más fiel en la letra a Marx, sólo el trabajo asalariado sería trabajo productivo. Esta visión ha sido puesta en cuestión desde el campo marxista feminista que ha defendido el carácter productivo del trabajo doméstico ya que produce una mercancía, en este caso la fuerza de trabajo, de manera que las mujeres están triplemente explotadas en el capitalismo; explotadas por los hombres en la estructura familiar, por el capital en cuanto amas de casa y explotadas en el proceso productivo si ejercen trabajo asalariado (Mies, 1998).

Según Fuchs, el trabajo de los prosumidores es también trabajo productivo por el mismo motivo. Aunque no sea trabajo asalariado está generando un valor y, por lo tanto, los prosumidores están siendo explotados. Ante quienes ponen en cuestión está explotación porque el usuario de redes sociales hace esa actividad con satisfacción y sin ser remunerado Fuchs utiliza dos argumentos. Por un lado, la tecnología constituye un velo que impide al prosumidor tomar conciencia de que está siendo explotado. Parece que las redes sociales benefician a sus usuarios, pero en realidad, en el mismo momento del uso, se está llevando a cabo un proceso de producción de una mercancía (Fuchs, 2014, p. 261). El segundo argumento plantea que existe coerción para que los usuarios trabajen produciendo datos, porque si el usuario de redes se niega a trabajar, es decir, a 
usar las redes, es privado de un servicio que se ha convertido en un elemento básico de articulación de las necesidades de comunicación de las personas. El primer argumento es muy recurrente en los estudios de fenómenos diversos en internet como mencionábamos anteriormente. El segundo es un argumento interesante porque nos plantea la posibilidad de que exista coerción tras una fachada de libertad de elección; pero no está necesariamente relacionado con la existencia de trabajo; puede existir coerción sin que exista trabajo en un sentido capitalista. En este caso, puede existir coerción para que el usuario ceda sus datos a la compañía que gestiona la plataforma.

Una visión alternativa es la que considera que las plataformas no obtienen los beneficios de la explotación laboral de los usuarios, sino que estarían obteniendo una renta por la monopolización de un recurso escaso, de un bien común, en este caso la información generada por el intelecto colectivo (Pasquinelli, 2009). Fuchs no comparte este punto de vista, porque si nos atenemos a una definición marxiana estricta de la renta esta sólo se genera a partir de aquellos activos como los recursos naturales que están sujetos a una escasez que proviene de límites físicos y que no pueden producirse a través del trabajo. Como la información puede producirse a través de trabajo, no sería un recurso natural y no generaría rentas y el beneficio provendría de la explotación del trabajo. Como veremos más adelante, la TVT no contempla que puedan existir monopolios en aquellos bienes que se producen a través de trabajo y no están sujetos a límites naturales porque parte de la idea de que en el capitalismo siempre existe competencia.

Llegados a este punto podríamos platearnos si es necesario considerar como trabajo la actividad que realizan los usuarios de redes sociales y que ventajas supone para el análisis y para la práctica política. La opción de Fuchs, reconocer la actividad en redes sociales como trabajo, se ve condicionada, como el mismo reconoce, por la voluntad de crear una unidad de acción política entre trabajadores de las plataformas y usuarios. Al ser dos colectivos que comparten la condición común de explotados, una condición situada además en la infraestructura, que para muchos marxistas es la base a partir de la cual se erige la superestructura cultural, ideológica y política; estos colectivos están llamados a desarrollar una conciencia de clase común que los articule en una lucha colectiva como sujeto revolucionario.

El problema aquí es que la aparición de una unidad política entre estos colectivos pasa más por que sean capaces de articular políticamente unas demandas comunes que por que ambos compartan la condición de explotados en base a la TVT. Como ha mostrado la historia social británica (Stedman, 1989; Thompson, 2012), la emergencia política de las clases sociales responde a procesos que se desarrollan en la arena política y que articulan a grupos que, en ciertos aspectos, pueden tener intereses divergentes. 


\section{Explotación, alienación y trabajo digital}

Para clarificar los procesos que se dan en torno al trabajo digital debemos partir de una definición lo más precisa posible de lo que es trabajo y lo que lo diferencia del consumo. Es cierto que no resulta sencillo separar las actividades de producción y consumo de una manera estricta. Como afirma Ritzer, en los procesos de producción se consumen materiales, maquinaria y trabajo humano. Sin embargo, si bien no podemos establecer una distinción estricta entre lo que es trabajo y lo que es consumo, si nos fijamos únicamente en la actividad en sí, esta distinción aparece mucho más clara si tenemos en cuenta la significación social de las actividades. El trabajo sería una manera de generar los recursos materiales e inmateriales necesarios para satisfacer las necesidades humanas. Realizamos trabajo porque existen una serie de necesidades sociales que requieren diferentes bienes y servicios para ser satisfechas y estos bienes y servicios necesitan ser producidos. Por lo tanto, cuando realizamos trabajo, no buscamos satisfacer nuestras necesidades en ese momento, sino que lo hacemos para obtener los recursos (productos o salario) que nos permitan satisfacer nuestras necesidades o las necesidades de otros, en un momento posterior ${ }^{1}$.

Es cierto que existen ciertas actividades en las que consumo y producción aparecen mezcladas, pero no serían tan numerosas como las que plantea Ritzer. Desde este punto de vista, el trabajo que se realiza en una fábrica, aunque implique el consumo de materiales, caería dentro de la esfera de la producción porque las personas trabajadoras no realizan ese trabajo porque satisfaga sus necesidades sino para obtener los ingresos necesarios para satisfacerlas por otros medios.

Ritzer parte de que la fusión entre producción y consumo en los procesos de prosumo es algo universal a todas las sociedades (Ritzer, 2014). Sin embargo, el uso de conceptos específicos para tratar de comprender el capitalismo puede resultar útil, ya que estamos ante un sistema económico que tiene como característica principal el hecho de que, bajo el capitalismo, el trabajo se convierte en una mercancía. Los procesos sociales que llevaron a la creación de los mercados de trabajo, además de generar importantes resistencias (Polanyi, 2016), tiende a separar el trabajo del resto de actividades sociales ${ }^{2}$.

La disciplina fabril encierra el proceso de trabajo ente cuatro paredes y posibilita que el capitalista organice la producción con el objetivo de maximizar la plusvalía que extrae de los trabajadores. Esta es la explotación que describe Marx en el vo-

\footnotetext{
${ }^{1}$ Como veremos más adelante, algunas de las estrategias de organización del trabajo que se han puesto en marcha en las últimas décadas tienen como objetivo principal aumentar la explotación a través de la presentación del trabajo como un fin en sí, capaz de satisfacer necesidades, en lugar de como un medio que nos permita obtener los recursos necesarios para poder satisfacer dichas necesidades.

2 Ritzer tiene en cuenta este proceso; pero plantea la emergencia del capitalismo prosumidor constituye una especie de eterno retorno nietzscheano a la situación previa a la revolución industrial.
} 
lumen I del capital. Sin embargo, la crisis del modelo fordista vuelve, a partir de los años ochenta, a romper las barreras de la fábrica y a extender el proceso de acumulación de capital hacia espacios sociales cada vez más indefinidos. En un momento en que el conocimiento, tal y como había previsto Marx, se convierte en una de las dimensiones fundamentales de la competencia (Fuchs, 2019), el control taylorista del proceso de producción empieza a mostrar sus límites. El proceso de control del trabajo inmaterial es difícil de materializar a través de las cadenas de producción de la fábrica fordista y requiere implementar nuevas tecnológicas organizativas que traten de controlar al trabajador no ya a través de mecanismos de coerción sino consiguiendo que interiorice los valores y los objetivos de la empresa. La teoría de las necesidades de Abraham Maslow (1991), la teoría X-Y de Douglas McGregor (1996), o la teoría Z de William Ouchi (1981) se convierten en referencias en las escuelas de negocios que tratan de generar ese nuevo espíritu del capitalismo (Boltanski y Chiapello, 2002) que empuja al trabajador a buscar la autorrealización a través del trabajo. Se trataría aquí de que el trabajador se convirtiera en su propio supervisor, de ganar la subjetividad del trabajador para la causa de la empresa.

Vemos aquí que fenómenos asociados a lo digital como la ludificación del trabajo no serían totalmente novedosos desde un punto de vista conceptual y entroncan perfectamente con las técnicas de organización de la empresa que se implantan a partir de los años ochenta como una forma de gestionar el trabajo inmaterial (Lazzarato, 1996) y, como una forma, también, de neutralizar lo que Boltanski y Chiapello denominan la crítica artista del sistema productivo (Boltanski y Chiapello, 2002). La crítica artista, vinculada a las revoluciones del 68, ponían en primer plano que los procesos productivos capitalistas eran alienantes porque impedían la autorrealización personal.

Deberíamos plantear, llegados a este punto, qué conceptos podría ser adecuados para dar cuenta de estas nuevas modalidades de trabajo que están tomando creciente importancia con el advenimiento de la era digital. Trataremos de hacer este trabajo teórico partiendo de algunos conceptos que tienen un largo recorrido dentro del pensamiento marxista; en concreto los conceptos de alienación y explotación. No se trata de conceptos que tengan una única acepción dentro del campo marxista, ni siquiera de la obra del propio Marx, por lo que vamos a tratar de clarificar el sentido que le daremos en este trabajo.

\subsection{Trabajo explotado}

Para Marx una persona es explotada si realiza una cantidad de trabajo mayor que el trabajo necesario para producir los bienes que consume. Para explicar cómo se produce la explotación bajo el sistema capitalista, un sistema en el que los individuos no son objeto de una coerción física directa para que trabajen, Marx desarrolla una teoría del valor- trabajo que a través del concepto de plusvalía explica como en el proceso productivo los capitalistas se apropian de parte del trabajo realizado por 
los trabajadores. La TVT es una herramienta analítica que trata de desvelar lo que el fetichismo de la mercancía mantiene oculto, que el capital no es una cosa sino una relación social a través de la cual el capitalista se apropia del trabajo del obrero.

Además de explicar este mecanismo de explotación, la TVT pretende explicar los precios de las mercancías en base al valor trabajo que llevan incorporado. Si bien en el caso del mecanismo de explotación del proceso productivo la teoría logra su objetivo, en el caso de la explicación de los precios los resultados son más limitados. La teoría del valor-trabajo (TVT) ha sido puesta en cuestión no solamente desde el campo liberal (Böhm-Bawerk, 1949), sino también desde el campo marxista. Desde el denominado marxismo analítico se han planteado teorías de la explotación que no están basadas en la TVT (Elster, 1991; Roemer, 1989) y que emplean herramientas de la economía ortodoxa para demostrar que la explotación en el capitalismo se produce también en condiciones de libre competencia.

En este trabajo se comparte esta visión crítica porque, para que la TVT nos sirva para explicar los precios de mercado, se tienen que dar condiciones de libre competencia que no son la norma en la economía actual. La existencia de intercambio desigual destruye las potencialidades de la TVT como teoría analítica. El intercambio desigual implica que existe una desviación sistemática de los precios respecto a los valores con lo cual los valores perderían gran parte de su capacidad explicativa de las dinámicas económicas visibles; es decir, de los precios.

La existencia de competencia en los mercados era un supuesto razonable en la época en la que Marx formula su teoría del valor. De hecho, Marx trataba de demostrar que en un sistema de libre competencia también se produce explotación. Sin embargo, en el capitalismo actual la TVT no serviría para explicar los precios por varios motivos. En primer lugar, la creciente concentración de capital a lo largo del último siglo y medio ha llevado a algunos autores marxistas a plantear que nos encontramos en una fase monopolista del capitalismo (Baran y Sweezy, 1988). Las innovaciones tecnológicas también crean monopolios temporales (Schumpeter, 1996) que permiten que los precios de los productos no se correspondan con la cantidad de trabajo incorporado. La estructura del comercio mundial dividida en países centrales y periféricos da lugar a que se produzca intercambio desigual que traslada el grueso de la explotación a los países periféricos (Emmanuel, 1980). Por último, la importancia del trabajo inmaterial (Lazzarato, 1996) y de mercados de trabajo segmentados (Doeringer y Piore, 1985) implica grandes diferencias salariales entre sectores que provocan, a su vez, fenómenos de intercambio desigual.

La existencia de competencia imperfecta implica, por lo tanto, que se puede dar intercambio desigual y, por lo tanto, explotación a través del mercado y no solamente en el proceso productivo a través de la extracción de la plusvalía. El intercambio desigual supone una transferencia de la plusvalía hacia la empresa que vende a un precio superior al valor-trabajo incorporado a su producto. 
Desde este punto de vista, aunque admitamos que es difícil explicar la estructura de precios en base a la TVT, se puede mantener, sin embargo, la TVT como base para una teoría normativa de la explotación que, según Elster, también está presente en la obra de Marx; a saber, que cada agente debería recibir medios de vida en proporción a la cantidad de trabajo que ha aportado es decir al valor que ha generado ${ }^{3}$.

Abandonar la TVT como herramienta analítica y considerarla únicamente como un referente normativo en base al cuál determinar los grados de explotación nos sirve también para superar la dicotomía trabajo productivo- trabajo improductivo. Desde este punto de vista, todo trabajo, como actividad orientada a generar los medios de vida necesarios para la satisfacción de necesidades humanas, es productivo, independientemente de que se haga para el mercado o para producir bienes para el autoconsumo. De esta manera, el trabajo doméstico sería tan productivo como el trabajo asalariado.

\subsection{Trabajo alienado}

John Elster distingue dos sentidos diferentes del concepto de alienación en Marx (Elster, 1991). En la obra temprana de Marx, la alienación aparece descrita como un estado psicológico. En sus Manuscritos económico - filosóficos Marx define la alienación como un sentimiento subjetivo de falta de control sobre el proceso de trabajo. Esta alienación subjetiva impide la autorrealización del individuo a través del proceso de trabajo, el individuo percibe que el proceso productivo no tiene sentido y que no tiene ninguna capacidad de control sobre el mismo.

Por el contrario, en El capital, que pertenece a la obra tardía de Marx, la alienación es una dimensión estructural; el trabajador está alienado respecto a los medios de producción, no es consciente de que el capital es trabajo muerto que no genera valor y es, por lo tanto, incapaz de ver que está siendo explotado, es incapaz de percibir que la ganancia del capitalista proviene del valor generado por su propio trabajo (el del obrero) ${ }^{4}$.

\footnotetext{
3 Elster plantea que esta teoría presenta también algunos problemas conceptuales, por ejemplo, el carácter heterogéneo del trabajo.

${ }^{4}$ La diferencia entre la obra temprana y madura de Marx fue también uno de los ejes del trabajo de Louis Althusser. Althusser considera que la primera etapa de Marx está muy influida por Hegel y que, en ella Marx no ha conseguido todavía salir de un esquema humanista que es, en gran medida, idealista. La noción de alienación subjetiva que hemos expuesto anteriormente se correspondería a este período de juventud de Marx. Por el contrario, Althusser considera que la postura del Marx maduro en El capital, rompe con esa concepción idealista e introduce un punto de vista estructural centrado en las relaciones de producción. En este contexto la ideología sería fruto de la posición social de los individuos, una entidad que los atraviesa y les impide ver la naturaleza real de las relaciones de producción en las que están insertos (Althusser, 1987).
} 
El primer concepto de alienación genera un malestar que impulsa la movilización del obrero contra el capital, el segundo tipo, por el contrario, dificulta dicha movilización porque oculta la naturaleza de la explotación (Elster, 1991, p. 127). Denominaremos al primer tipo de alienación, alienación subjetiva y al segundo alienación objetiva.

La que Boltanski y Chiapello denominan crítica artista del capitalismo combate la alienación subjetiva, es decir, la alienación entendida en el sentido en el que la entendía el joven Marx. La crítica del proceso de trabajo como proceso alienante que se articula a partir de las revoluciones de 68 y de trabajos como los de la escuela de Frankfurt, va a generar innovaciones en los modos de organización del trabajo que van a conseguir darle la vuelta a la crítica, asimilarla y utilizarla para hacer frente a uno de los problemas de la organización del trabajo en el contexto de un nuevo capitalismo cognitivo o informacional; a saber, como hacer frente al problema del control del trabajo intelectual que no se presta a ser controlado por los métodos tayloristas tradicionales.

Es un debate interesante el que se genera en este punto. ¿Está realmente alienada una persona que realiza un trabajo bajo el impulso de su propia motivación sin necesidad de que existan mecanismos de coerción? Desde el punto de vista de la alienación subjetiva no; el trabajador percibiría sentido en el trabajo que realiza y además tendría un mayor control sobre el mismo, por lo tanto, su alienación subjetiva disminuiría. Sin embargo, desde el punto de vista de la alienación objetiva, tenemos que el trabajador es menos consciente de que está siendo explotado; ni siquiera sería necesaria la coerción para conseguir extraer la plusvalía del trabajador porque éste ha asumido las motivaciones ligadas al proceso de acumulación como motivaciones propias. Estas son condiciones más propicias para que la explotación sea mayor. Vemos, por lo tanto, que, bajo ciertas circunstancias, la alineación subjetiva y la objetiva están negativamente correlacionadas.

La alienación subjetiva genera rechazo y hace que el trabajador tienda a rebelarse contra la organización del proceso productivo, trabajando lo mínimo posible en ausencia de control, exigiendo una mayor remuneración, etc. Esto disminuye la alienación objetiva porque en esas circunstancias el trabajador estará más dispuesto a articular reivindicaciones respecto al salario o las condiciones de trabajo y, por lo tanto, es más consciente de que está siendo objeto de explotación.

Un modo de organización del trabajo que consiga reducir la alienación subjetiva, por el contrario, aumentará la motivación del trabajador, reducirá su grado de resistencia ante el proceso de productivo y probablemente consiga establecer un mayor grado de explotación.

En definitiva, tenemos que la alienación subjetiva tiende a estar negativamente correlacionada con el grado de explotación mientras que la alienación 
objetiva tiende a estarlo positivamente. Las técnicas de organización de recursos humanos de las que hablábamos anteriormente (Maslow, Ouchi o McGregor) van orientadas a que los trabajadores encuentren satisfactores (MaxNeef, 1993) en el desarrollo de sus labores y, de esta manera, aumenten su motivación (reduzca su alienación subjetiva) y estén dispuestos a que se les explote más.

\section{Figura 1}

Diagrama de la relación entre explotación y alienación subjetiva

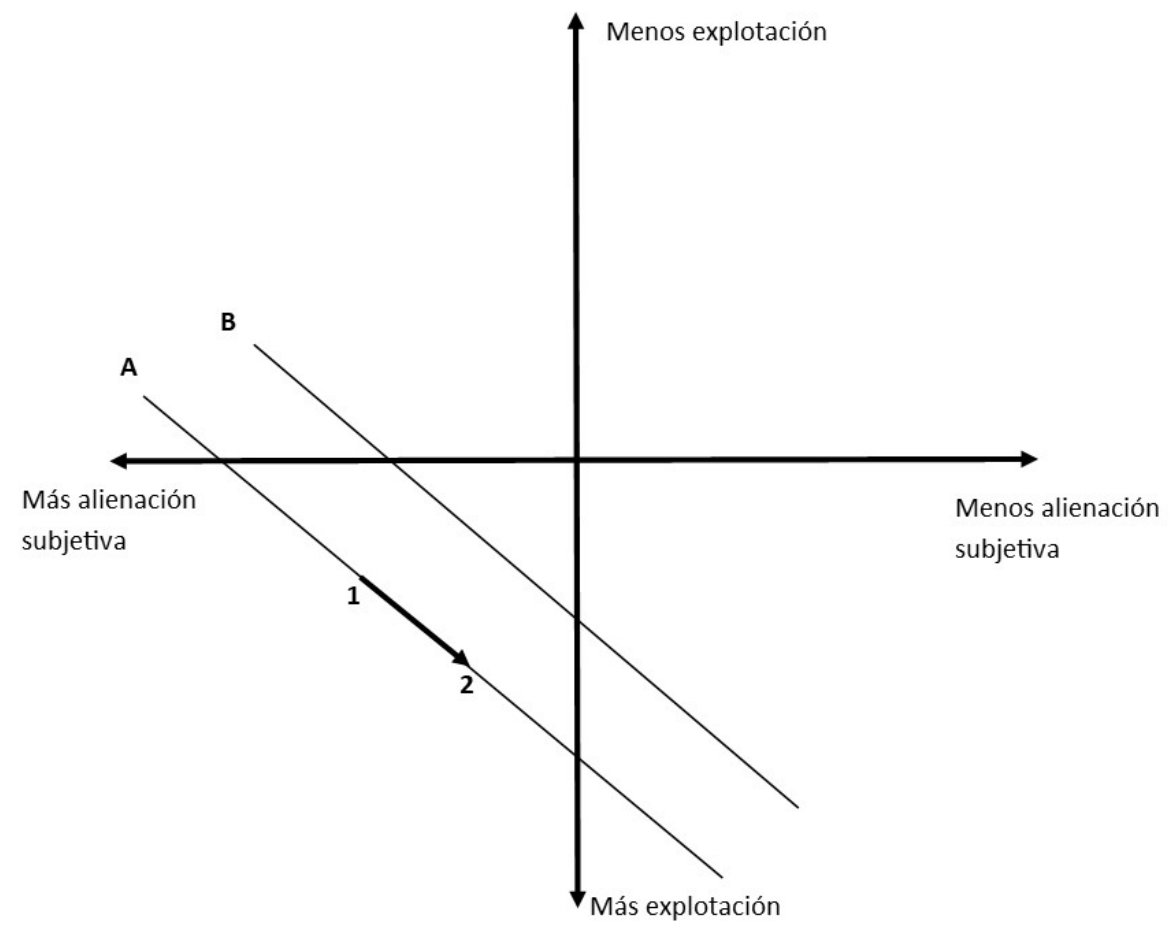

Fuente: elaboración propia.

La figura 1 trata de recoger esta hipótesis de relación entre alienación subjetiva y explotación. Es importante resaltar aquí que no debemos entender la condición alienado-no alienado como una dicotomía, sino más bien, como un continuo; se puede estar más o menos alienado. De la misma manera, se puede estar más o menos explotado dependiendo de que la tasa de plusvalía sea mayor o menor. 
Las dos rectas A y B serían rectas isobienestar. Todos los puntos de una recta isobienestar proporcionan la misma satisfacción al trabajador. Si comparamos los puntos 1 y 2 vemos que en el punto 1 el trabajador se encuentra en un mayor grado de alienación subjetiva, pero está menos explotado. Si se introducen técnicas del trabajo que traten de aumentar la motivación del trabajador y éste le empieza a encontrar sentido al trabajo, empieza a identificar sus intereses con los de la empresa, es probable que el trabajador esté dispuesto a trabajar más y a intensificar el trabajo a pesar de que no le suban el salario. Pasaría de esta manera del punto 1 al 2, donde teniendo el mismo grado de satisfacción, está siendo más explotado y tiene un menor grado de alienación subjetiva.

\section{Una tipología del trabajo (y del no trabajo) en el capitalismo digital}

Partiendo de lo expuesto en el anterior apartado vamos a ver como se articulan las dimensiones de la alienación y de la explotación en los nuevos trabajos que surgen en torno a la automatización y digitalización de los procesos de producción y consumo. Partimos de una tipología que no pretende ser exhaustiva; existen muchos otros trabajos relacionados con el capitalismo digital. Algunos de ellos están recogidos en la tipología de Fuchs (Fuchs, 2014) expuesta anteriormente. Nos centramos aquí en actividades que presentan algún grado de novedad en su configuración como proceso de trabajo, en concreto, aquellas actividades que podrían ser susceptibles de encajar bajo la categoría de prosumo. Así, dejamos de lado trabajos como la producción de hardware que, a pesar de producir productos novedosos relacionados con lo digital, mantiene unos procesos de trabajo similares a los de los esquemas tayloristas (Fuchs, 2014).

Nos hemos centrado en actividades que se realizan sobre todo en países del centro, aunque muchos de estos trabajos ya se están dando o se irán dando en los próximos ańos en los países periféricos. Los cuatros tipo de trabajo digital que vamos a analizar son: el autoservicio en los procesos de compra, la generación de datos por parte de los usuarios en las redes sociales, el caso de los trabajadores uberizados y los creadores de contenidos para plataformas digitales.

\subsection{Autoservicios y más: cómo poner a los consumidores a trabajar}

Lo que se ha venido a llamar "poner a los consumidores a trabajar" es una técnica de reducción de costes que no es exclusiva de los negocios digitales, pero a la que el crecimiento del comercio digital ha dado un impulso significativo. Como apuntaba Ritzer (2015b) empresas como IKEA trasladan parte de sus procesos de producción al consumidor: el consumidor tiene que buscar los productos en la tienda, bajar al almacén, cargarlos, llevarlos a su domicilio y finalmente montarlos. Los costes laborales que implica el proceso de producción de 
un mueble se reducen de forma significativa. El caso de los portales de compra en internet, como Amazon, suponen también un ahorro importante de trabajo para las compañías que no tiene que desarrollar muchas de las actividades de exposición del producto, reposición y almacenamiento y acumulación de stocks que realizaban anteriormente.

Desde un punto de vista liberal marginalista, si los consumidores aceptan realizar todos estos trabajos es porque se están viendo compensados de alguna manera; en caso contrario la competencia beneficiaría a la empresa que no pusiese a trabajar a los consumidores. El punto de vista de Ritzer es, como comentábamos anteriormente, que estos prosumidores están siendo explotados porque desconocen los costes que se ahorra la empresa por esta vía y, por lo tanto, son incapaces de negociar una reducción de precios que justifique el ahorro de trabajo por parte de la empresa. Estaríamos ante algo muy similar a la alienación objetiva de la que hablábamos anteriormente; se crea un velo que impide ver que el valor del producto se está generando en parte por el trabajo del propio prosumidor y no por la empresa.

En este punto surge una duda. ¿es el concepto de prosumidor suficiente para explicar el fondo del asunto o solamente nos aporta una función descriptiva del tipo «el consumidor también aporta trabajo»? Por otro lado, tampoco parece que el fenómeno sea nuevo. En gran cantidad de productos -léase los alimentos, por ejemplo -, el consumidor tiene que realizar trabajo en el ámbito doméstico (cocinar) para poder consumir el producto.

La lucha social para que el ámbito doméstico asuma una parte creciente del trabajo necesario para reproducir la sociedad ha sido, de hecho, el tema central de la economía feminista marxista (Federici, 2014; Mies, 1998). Tenemos, por lo tanto, un aparato teórico que quizás nos permita comprender mejor el fenómeno que el propio concepto de capitalismo de prosumidores. Es relevante en este sentido también la aportación realizada por Gershuny y Pahl (1979) donde ya proponían que en la década de los setenta se estaba produciendo un importante traslado del trabajo al ámbito doméstico, cómo las familias adquirían crecientemente bienes de capital (electrodomésticos) para realizar trabajo doméstico en lugar de adquirir servicios domésticos en el mercado5.

Así, los procesos de compra podrían enclavarse dentro de los trabajos domésticos. En este sentido el crecimiento de actividades de autoservicio enfocadas a poner a los consumidores a trabajar tendría como objetivo cargar parte del tra-

5 Estos autores platearon también que se estaba produciendo un aumento de la economía sumergida como consecuencia entre otras cosas de un mercado laboral crecientemente inseguro que condenaba al desempleo a una parte importante de las personas activas. El papel de la precariedad y el desempleo crónico en el aumento de los empleos de falso autónomo de los que se nutren las plataformas tampoco debería ser desatendido. 
bajo de mercado de las empresas sobre las espaldas del trabajo doméstico. Ritzer explica bien el proceso de implementación de estos sistemas por parte de las empresas en el marco de una teoría de la racionalización inspirada por Max Weber (Ritzer, 1996). Sin embargo, esta teoría explica por qué las empresas aplican estos procesos, pero no por que los consumidores los aceptan sin resistencias.

El concepto de alienación nos podría resultar útil para dar una explicación. La mercadotecnia no sólo crea deseo respecto a un producto también lo puede crear en torno a una marca, una empresa e, incluso, en torno al procedimiento de compra. Si consideramos el proceso de compra como parte del trabajo doméstico, las técnicas de ludificación o la publicidad pueden hacer aparecer este trabajo como algo que tenga sentido para el consumidor, más allá de que el trabajo sea necesario para consumir el producto. A pesar de que el trabajo doméstico es un trabajo que implica, en principio, una alienación menor que el trabajo asalariado en una empresa, estas técnicas pueden contribuir, a través de la aprehensión de la subjetividad del consumidor, a reducir la alienación subjetiva, a aumentar la alienación objetiva (el trabajador pierde la noción de que está realizando un trabajo) y, por lo tanto, a aumentar la explotación, es decir, a aumentar la cantidad de trabajo que el consumidor está dispuesto a realizar sin remuneración ni disminución de precio.

\subsection{Usuarios de plataforma: la mercantilización de la privacidad}

Desde la concepción del trabajo que hemos expuesto en el apartado anterior, los usuarios de redes sociales (en tiempo de ocio) no estarían realizando un trabajo sino consumiendo. La actividad que realizan no se hace como un medio para satisfacer las necesidades propias o ajenas, sino porque la propia actividad satisface las necesidades, de comunicación o entretenimiento, de la persona que la realiza. La actividad de consumo contribuye a la producción de una mercancía, en este caso los datos, de un modo similar a como el llevar una camiseta de marca por la calle contribuye a hacer publicidad para una empresa. El proceso de generación de datos requiere trabajo, pero este trabajo se da dentro de la propia compañía e implicaría todas las tareas necesarias para que la plataforma esté lista para ser usada.

En cualquier caso, lo que sí que está haciendo el usuario, consciente o inconscientemente, es mercantilizar su privacidad, pagando con sus propios datos por el derecho a usar un servicio informático. Algunos autores han planteado que este proceso tiene similitudes con los procesos de privatización y mercantilización de los bienes comunes que se dieron en los inicios del capitalismo industrial. En este caso el bien común sería un intelecto general, todo el conjunto de la información generada por la humanidad en sus interacciones comunicativas, que es apropiado por las plataformas que lo utilizan para generar rentas (Pasquinelli, 2009). 
La pretensión de Fuchs de definir la actividad en redes sociales como trabajo tiene como objetivo unir a usuarios y trabajadores de las plataformas en una lucha revolucionaria que destruya el poder de estas grandes empresas. Para ello, como buen marxista que piensa que la explotación compartida lleva a una conciencia de clase compartida y, ésta, a la movilización política, necesita que usuarios y trabajadores estén siendo explotados en los términos de la TVT.

Sin embargo, la conciencia de la explotación no es el único resorte que puede activar una movilización política contra estas corporaciones. De hecho, en términos puramente pecuniarios el usuario puede ver el intercambio como un proceso simbiótico: la empresa gana dinero con mis datos, pero yo gano el uso gratuito de un servicio. Incluso aunque consideremos que el usuario está trabajando, si ello no le supone ningún tiempo ni esfuerzo y le reporta un beneficio - el uso gratuito del servicio-, no parece que su supuesta condición de explotado teórico en base a la TVT vaya a empujarle a rebelarse contra esa situación.

Sin embargo, si consideramos el proceso de producción de datos como un proceso que, por un lado, al mercantilizarla, pone en riesgo la privacidad de los usuarios y, por otro lado, está contribuyendo a la privatización, o la acumulación por desposesión (Harvey, 2007) de un bien común, el intelecto general; tenemos que las posibilidades de movilización fuera de la lógica de la TVT no son para nada desdeńables. Los usuarios pueden movilizarse para defender su derecho individual a la privacidad y también pueden movilizarse para defender el derecho colectivo al conocimiento. Pueden movilizarse también para evitar que la lógica mercantil impregne todo un espacio de interacciones, internet, que se ha convertido en un espacio central para la comunicación humana, y para la actividad humana en general. En lo que algunos autores han denominado capitalismo de la vigilancia (Zuboff, 2019), la mayoría de las comunicaciones de internet deben hacerse por medio de plataformas privadas que monitorean la privacidad, generando un capital cultural objetivado privado (los datos) que les otorga importantes ventajas en numerosos campos sociales (Barba del Horno, 2020). La recuperación de ese espacio social como espacio público es un proyecto en el que no solamente pueden coincidir usuarios y trabajadores de las redes sociales sino mayorías sociales amplísimas.

Vemos pues, que tenemos numerosas herramientas y conceptos para comprender estos procesos nuevos, por lo que considerar a los usuarios de las redes sociales como prosumidores explotados en base a la TVT puede oscurecer el problema de fondo en lugar de aportar luz para hacerle frente.

\subsection{Trabajadores uberizados: control digital mediante la plataforma}

Uno de los temas relacionados con el trabajo digital que más de actualidad ha estado en los últimos tiempos es el de los trabajadores de plataformas de lo 
que, en ocasiones ha sido denominado como trabajo colaborativo. Estamos hablando de los trabajadores de empresas como uber, just eat, glovo o deliveroo, cuya forma de organizar el trabajo está provocando transformaciones significativas en el terreno laboral (Rosenblat, 2018). En este caso, uno de los problemas a nivel jurídico ha sido el de la contratación de trabajadores dependientes en fraude de ley como falsos autónomos. En este caso, el problema se deriva de que la novedad de la gestión del trabajo a través de plataformas ocultaba parcialmente el carácter dependiente de los trabajadores. Como las labores de control estaban programadas a través de algoritmos eran menos visibles, lo que llevó a las empresas a tratar de no reconocer a sus trabajadores como asalariados y privarles, por lo tanto, de toda la protección que ofrece el derecho laboral.

Se ha planteado aquí también la función de ocultamiento del algoritmo, que velaría el proceso de explotación del trabajo. Una vez más no parece que este argumento sea demasiado convincente. Al igual que en el caso de los perceptores de ayudas sociales (Eubanks, 2018), los afectados por los algoritmos, en este caso los trabajadores uberizados, son los primeros en darse cuenta de su funcionamiento, deduciéndolo de sus efectos.

Al margen de este problema derivado de la indefinición jurídica de las actividades y de un proceso más amplio de terciarización que se remonta varias décadas atrás, deberíamos preguntarnos en qué medida transforman el trabajo estos procesos de automatización digital. Si nos atenemos a la transformación sobre los empleos vemos que lo que ahorra fundamentalmente es trabajo de control. Las labores de control se trasladan a una aplicación que tiene además la virtud de ser ubicua. De ahí que la organización y supervisión del trabajo en base a algoritmos haya sido importante, sobre todo, en trabajos de reparto donde el control de los trabajadores es complicado con los métodos tradicionales de supervisión.

En cuanto a las características de los empleos uberizados, se trata predominantemente de empleos que no requieren cualificación y que soportan grandes niveles de explotación y de alienación subjetiva (Glavin et al., 2020), circunstancias exacerbadas por el hecho de que los trabajadores están solos la práctica totalidad del tiempo de trabajo y mantienen poco o nulo contacto con sus compañeros. Cabría preguntarse en qué medida la uberización puede contribuir a una mayor descualificación profesional (Braverman, 1975), lo que reduce el poder de negociación de los trabajadores, y en qué medida la ludificación de este tipo de trabajos puede contribuir a una reducción de la alienación subjetiva y, por lo tanto, a una mayor explotación.

\subsection{Trabajo autónomo digital: entre la autoexplotación y el éxito profesional}

Por último, tendríamos el caso de los creadores de contenidos para las plataformas online. En este caso y a diferencia de los usuarios de redes sociales 
existe una vocación de realizar un trabajo de forma profesional o de realizarlo de forma no remunerada o parcialmente remunerada, pero con la intención de generar contenidos que son puestos a disposición del público.

Se trata de una actividad que los usuarios suelen desarrollar, en ocasiones, con gusto y con un alto grado de autonomía por lo que cabría decir que implica bajos niveles de alienación subjetiva. Esta circunstancia provoca que los creadores de contenidos puedan invertir mucho tiempo y recibir, a cambio una remuneración mínima o nula. Se plantearía aquí la cuestión de cuál es el grado de explotación al que están sujetos. En este caso podemos encontrarnos situaciones muy diversas, desde una minoría de trabajadores que obtienen grandes ingresos, hasta una mayoría que están en condiciones extremadamente precarias desde el punto de vista de la estabilidad y cantidad de los ingresos que reciben.

En cualquier caso, si tuviéramos que definir una tendencia general en este ámbito de trabajo, cabría apuntar que las plataformas han abierto espacios crecientes para el trabajo autónomo con un bajo nivel de alienación subjetiva. Los creadores tienen más libertad que en el pasado para poner sus creaciones en manos del público y más libertad para definir sus propias creaciones; sin embargo, esto ha dado lugar a una mayor explotación en la mayoría de los casos y a una mayor alienación objetiva; pues al situarse el trabajo bajo una relación mercantil y no asalariada resulta más complicado percibir la dimensión de la explotación que esconde.

Tampoco son descartables los efectos que la competitividad entre diferentes creadores tiene en la autoexplotación. En este sentido, es interesante también recuperar el planteamiento de la carrera de ratas planteado por Akerlof (1984), para el caso de los trabajadores sujetos a procesos de promoción interna en las empresas. La competitividad para obtener un ascenso y un salario más elevado lleva, en ocasiones, a que los trabajadores aumenten su productividad por encima de los aumentos salariales asociados a la promoción, generando de esta manera, unos mayores ingresos para la empresa y aumentando su nivel de explotación.

\subsection{Alienados y explotados: posiciones relativas de los trabajadores digitales}

En la Figura 2 se resumen las características en cuanto a las dimensiones alienación y explotación de los diferentes tipos de trabajos digitales descritos en los epígrafes anteriores. Hemos añadido, además, las categorías «trabajadores cualificados fordistas" $\mathrm{y}$ "trabajadores no cualificados fordistas» como puntos de comparación. Los trabajadores cualificados fordistas se encontrarían en una situación asalariada tradicional, con un alto nivel de alienación subjetiva, poca 
autonomía para definir su trabajo, pero unas condiciones laborales ventajosas, derivadas de su condición asalariada y cualificada, que implicarían una menor explotación. Los trabajadores no cualificados fordistas serían objeto de una mayor explotación que los cualificados y su grado de alienación sería también mayor por tener un menor grado de control sobre el proceso de trabajo.

Se puede apreciar, si comparamos los nuevos empleos con los tradicionales, que existiría una tendencia, a nivel teórico, a que los procesos de digitalización del trabajo puedan dar lugar a una mayor explotación a través de la promesa de una menor alienación subjetiva, una mayor autonomía y un mayor control sobre el proceso de trabajo. Los trabajos digitales también aumentan la explotación a través de una mayor alienación objetiva al generar un marco en el que las relaciones explotador-explotado son más difusas, por darse muchas de ellas a través de mecanismos de intercambio, y por una creciente separación física de la masa de trabajadores.

Figura 2

Trabajadores digitales en el diagrama explotación-alienación

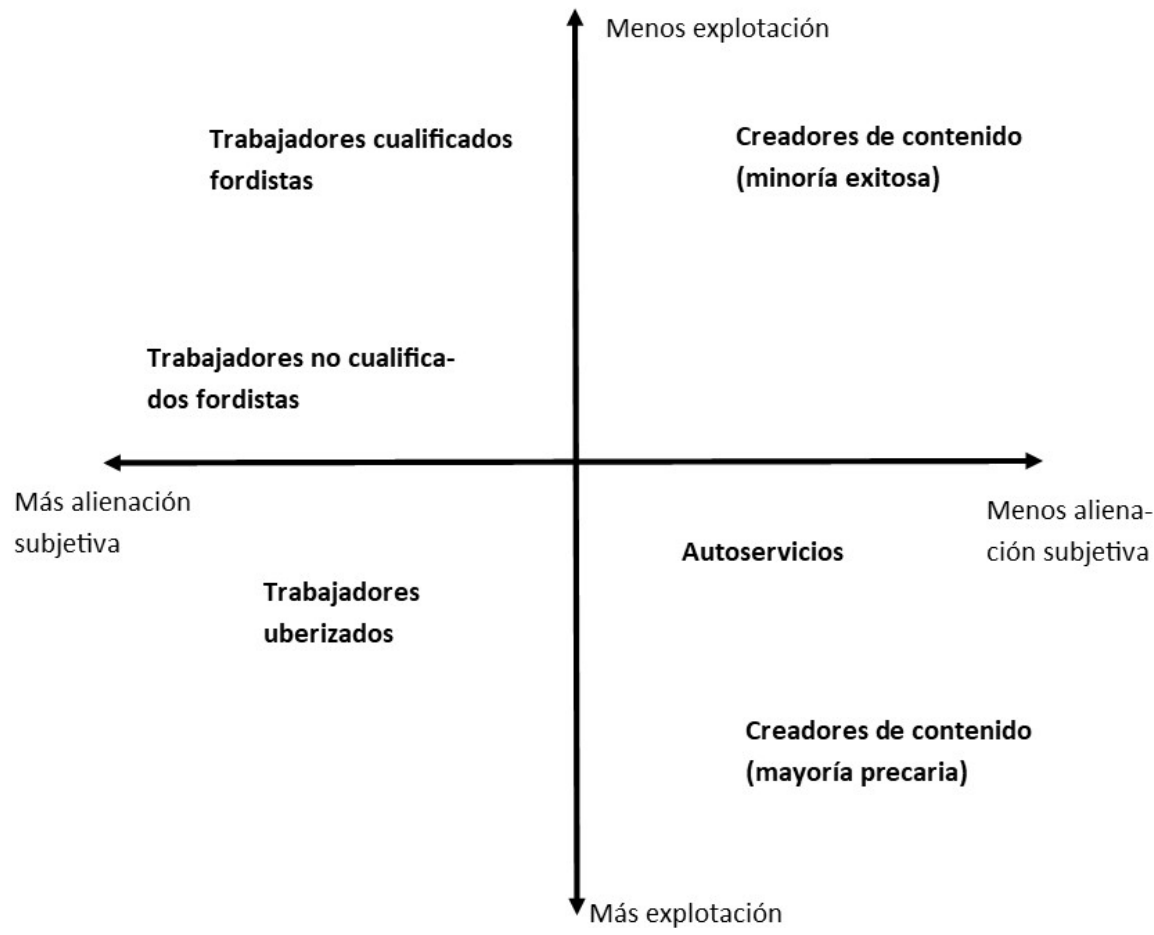

Fuente: elaboración propia. 


\section{Conclusiones}

A lo largo del artículo hemos defendido la idea de que se puede hacer frente al reto de explicar las nuevas dinámicas del trabajo digital en base a conceptos y teorías de larga tradición en las ciencias sociales. La producción de teorías y conceptos excesivamente rupturistas puede hacernos caer en cierto epocalismo y puede oscurecer, en último término, la comprensión de la realidad. Concretamente hemos puesto en cuestión la capacidad explicativa de un concepto, el de prosumidor, que pensamos que tiene un carácter más descriptivo que analítico.

A pesar de que la aparición de internet ha generado transformaciones importantes en muchas de las dinámicas del trabajo pensamos que es exagerado, al menos de momento, decir que hemos entrado en una nueva fase del capitalismo con lógicas diferentes a las que venían dándose en las décadas anteriores. Hemos tratado de explicar que las transformaciones del trabajo digital entroncan con unos procesos que se venían produciendo desde los años setenta y que se han caracterizado por una estrategia del capital de intensificación de la explotación a través de estrategias que tratan de reducir la alienación subjetiva.

\section{Bibliografía}

Akerlof, George A. (1984). An Economic Theorist's Book of Tales. Cambridge University Press.

Althusser, L. (1987). Ideologia y aparatos ideológicos del estado. Ediciones Quinto Sol.

Baran, Paul y Sweezy, Paul. (1988). El capital monopolista: Ensayo sobre el orden económico y social de Estados Unidos. siglo XXI.

Barba del Horno, M. (2020). «Campo, conexión y desigualdad. Hacia una economía política de las prácticas en la era del capitalismo digital». Teknokultura, 17(2), 121-130.

BECK, Ulrich (2001). «Interview with Ulrich Beck». Journal of Consumer Culture, 1(2), 261-277. https: //doi.org/10.1177/146954050100100209

BöHm-Bawerk, Eugene von (1949). Karl Marx and the Close of His System. Ludwig von Mises Institute.

Boltanski, Luc y Chiapello, Éve (2002). El nuevo espíritu del capitalismo. Ediciones AKAL.

Braverman, Harry (1975). Trabajo y capital monopolista: La degradacion del trabajo en el siglo XX. Nuestro Tiempo.

Doeringer, Peter y Piore, Michael. (1985). Mercados Internos de Trabajo y Analisis Laboral. Ministerio de Trabajo y Asuntos Sociales. Trabajo.

ELSTER, John (1991). Una introducción a Karl Marx. siglo XXI de Espańa.

EMmanuel, Arghiri (1980). Imperialismo y comercio internacional: El intercambio desigual. siglo XXI.

Eubanks, Virginia (2018). Automating Inequality: How High-Tech Tools Profile, Police, and Punish the Poor. St. Martin's Publishing Group.

Federici, Silvia (2014). La inacabada revolución feminista: Mujeres, reproducción social y lucha por la común. Ediciones Desde Abajo. 
Fuchs, Christian (2014). Digital Labour and Karl Marx. Routledge.

Fuchs, Christian (2015). "The Digital Labour Theory of Value and Karl Marx in the Age of Facebook, YouTube, Twitter, and Weibo», en Fisher, Eran y Fuchs, Christian (eds.), Reconsidering Value and Labour in the Digital Age (pp. 26-41). Palgrave Macmillan UK. https: //doi.org/10.1057/9781137478573_2

Fuchs, Christian (2019). Rereading Marx in the Age of Digital Capitalism. Pluto Press.

Gershuny, Jonathan I., y Pahl, Ray E. (1979). "Work outside employment: Some preliminary speculations». Higher Education Quarterly, 34(1), 120-135. https: //doi. org/10.1111/j.1468-2273.1979.tb01252.x

Glavin, Paul, Bierman, Alex y Schieman, S.Scott (2020). Über-Alienated: Powerless and Alone in the Gig Economy. Disponible en : https: //www.researchgate.net/ publication/343553987_Uber-Alienated_Powerless_and_Alone_in_the_Gig_Economy

Harvey, David (2007). Breve historia del neoliberalismo. Ediciones AKAL.

Lazzarato, Maurizio (1996). «Immaterial Labour», en Hardt, Michael y Virno, Paolo (eds.) Radical Thougth in Italy;: A Potential Politics (pp. 133-147). University of minessota Press.

Lipovetsky, Gilles (2010). La felicidad paradójica: Ensayo sobre la sociedad de hiperconsumo. Editorial Anagrama S.A.

Marx, Karl (1981). El capital: Critica de la economía politica. siglo XxI.

Maslow, Abraham (1991). Motivación y personalidad. Ediciones Díaz de Santos.

MaX-Neef, Manfred (1993). Desarrollo a escala humana. Icaria Antrazyt. http: //www. agapea.com/libros/Desarrollo-a-escala-humana-isbn-8474262178-i.htm

McGregor, Douglas (1996). El lado humano de las organizaciones. McGraw Hill.

Mies, Maria (1998). Patriarchy and Accumulation on a World Scale: Women in the International Division of Labour. Palgrave Macmillan.

Morozov, Evgeny (2012). The Net Delusion: The Dark Side of Internet Freedom. Hachette UK.

OIT. (2019). LAS PLATAFORMAS DIGITALES Y EL FUTURO DEL TRABAJO: CÓMO FOMENTAR EL TRABAJO DECENTE EN EL MUNDO DIGITAL.

Ouchi, William (1981). Teoria Z: Como pueden las empresas hacer frente al desafafio japones. Norma.

Pasquale, Frank (2015). The Black Box Society. Harvard University Press.

Pasquinelli, Matteo (2009). "Google's PageRank Algorithm: A Diagram of the Cognitive Capitalism and the Rentier of the Common Intellect». Deep Search.

Polanyi, Karl (2016). La gran transformación: Crítica del liberalismo económico. Virus Editorial.

Ritzer, Geroge (1996). La McDonalización de la sociedad: Un análisis de la racionalización en la vida cotidiana. Ariel.

Ritzer, George (2014). «Prosumption: Evolution, revolution, or eternal return of the same?» Journal of Consumer Culture, 14(1), 3-24. https: //doi.org/10.1177/1469540513509641

Ritzer, George (2015a). "The «New» World of Prosumption: Evolution, «Return of the Same,» or Revolution?», Sociological Forum, 30(1), 1-17. https: //doi.org/10.1111/socf. 12142

Ritzer, George (2015b). «Prosumer Capitalism». The Sociological Quarterly, 56(3), 413445. https: //doi.org/10.1111/tsq.12105

Roemer, John E. (1989). Teoría general de la explotación y de las clases. siglo XXI de España.

Rosenblat, Alexandra (2018). Uberland: How Algorithms Are Rewriting the Rules of Work. Univ of California Press. 
Schumpeter, Joseph A. (1996). Teoría del desenvolvimiento económico: Una investigación sobre ganancias, capital, crédito, interés y ciclo económico. Fondo de Cultura Económica.

Stedman Jones, Gareth. (1989). Lenguajes de clase: Estudios sobre la historia de la clase obrera inglesa (1832-1982). siglo XXI.

Thompson, Edward P. (2012). La formación de la clase obrera en Inglaterra. Capitán Swing.

TOFFler, Alvin (1985). La tercera ola. Orbis.

Zuboff, Shoshana (2019). The Age of Surveillance Capitalism: The Fight for a Human Future at the New Frontier of Power: Barack Obama's Books of 2019. Profile Books. 